\title{
Date and Time
}

National Cancer Institute

\section{Source}

National Cancer Institute. Date and Time. NCI Thesaurus. Code C37939.

An expression of both date and time that an event has happened or will happen. 